\title{
Deuda social con la educación y formación de jóvenes y adultos: accesibilidad, barreras y escenarios complejos
}

Dívida social com a educação e formação de jovens e adultos: acessibilidade,
barreiras e cenários complexos
Social educational debt and youth and adult training: acessibility, barriers and
complex backgrounds

GRACIELA CLOTILDE RIQUELME

NATALIA HERGER

ALEXANDER IVAN KODRIC

\begin{abstract}
Resumen: Esta ponencia tiene por objeto una revisión de la noción de derecho a la educación, desde una perspectiva de la política y planificación de la educación de jóvenes y adultos. La noción de deuda social educativa expresa el no cumplimiento de ese derecho por parte del Estado para con sus ciudadanos. Este artículo corresponde a una investigación cuyo objetivo general es contribuir a la comprensión de la diversificación y superposición de las ofertas de educación y formación para el trabajo y evaluar la dimensión de la deuda social educativa en ella involucrada. El encuadre conceptual articula los niveles macro, meso y micro y cuanti-cualitativo en la interpretación de las políticas públicas y los problemas de coordinación intersectorial.
\end{abstract}

Palabras clave: Derecho a la educación; Deuda social educativa; Jóvenes y adultos.

Resumo: Esta comunicação tem por objetivo uma revisão da noção de direito à educação, desde uma perspectiva da política e planejamento da educação de jovens e adultos. A noção de dívida social educativa expressa o não cumprimento desse direito por parte do Estado para com os seus cidadãos. Este artigo corresponde a uma pesquisa cujo objetivo geral é contribuir para a compreensão da diversificação e superposição das ofertas de educação e formação para o trabalho e avaliar a dimensão da dívida social educativa nela involucrada. O enquadre conceitual articula os níveis macro, meso e micro e quanti-qualitativo na interpretação das políticas públicas e os problemas de coordenação intersetorial.

Palavras-chave: Direito à educação; Dívida social educativa; Jóvens e adultos.

Abstract: This paper aims at reviewing the notion of the right to education from a political perspective and educational planning of youth and adults. The notion of educational social debt expresses the non-fulfillment of this right by the State towards its citizens. This article corresponds to an investigation whose general objective is to contribute to the understanding of the diversification and 
overlapping of the education offerings and training for the work and to evaluate the dimension of the educational social debt involved in it. The conceptual framework articulates the macro, meso and micro, and quantitative-qualitative levels in the interpretation of public policies and the problems of intersectoral coordination.

Keywords: right to education; social educational debt; youths and adults.

\section{INTRODUÇÃO}

La Argentina del 2001 puso al descubierto las necesidades sociales en medio de una profunda crisis política, social y económica que posibilitó la movilización de sectores, clases y grupos sociales para exigir la atención de demandas contrapuestas: desde la población indigente, la doblemente excluida del empleo y bienes sociales con niveles crecientes de pobreza.

Sostuvimos que

el incremento de la pobreza y la exclusión social comenzaron a incidir en esa población, tanto para los que accedían a la escuela, como en las dificultades del trabajo escolar y para los excluidos del sistema educativo. Al decir de algunos, el sistema educativo acrecentó la existencia de circuitos: unos para la inclusión y otros para la exclusión. La distribución desigual de la educación había permitido durante el siglo veinte un cierto ascenso social, ya que no había tanta concentración de ingresos y la educación fue siempre considerado un bien de acceso generalizado en el estado de bienestar, que permitía dicho desplazamiento inter-clase social (Riquelme, 2007, p.10).

La educación y formación para el trabajo constituye un derecho social de los jóvenes y adultos, que debe asentarse en los logros de la educación básica, primaria y secundaria, base de todo "proyecto educativo" (Bélanger y Federighi, 2004). Es por ello que se sostiene la prioridad del acceso y completitud de la educación primaria y secundaria con contenidos relevantes y mayores niveles de logro, sobre la que se asienten los futuros aprendizajes en los otros niveles del sistema, así como la realización de cursos de educación y formación para el trabajo.

La obligatoriedad del nivel primario y secundario, reconocida en la última década por la legislación nacional y de muchos países de América Latina, instala con mayor fuerza la responsabilidad del Estado en garantizar la oferta de servicios educativos a todos aquellos que no han completado su escolaridad. Para acompañar esta normativa, las políticas de ingresos condicionados (Asignación Universal por Hijo, Progresar, entre las más recientes) sin duda constituyen intervenciones que estimulan a la población excluida con un acompañamiento a 
jóvenes y adultos; a la vez determinan un fuerte impacto en el sistema educativo, con contradicciones y aspectos positivos que merecen ser evaluados.

Este artículo presenta una investigación ${ }^{1}$ cuyo objetivo general es contribuir a la comprensión de la complejidad, diversificación y superposición de ofertas de educación y formación para el trabajo y evaluar la dimensión de la deuda social educativa en ella involucrada y la estimación de los recursos individuales y sociales aplicados y no apropiados por diferentes ámbitos de la política educativa y social (trabajo, desarrollo social y salud). El objetivo es identificar factores que intervienen en la regulación de las políticas a nivel del Estado nacional, provincial, regional y local que favorecen la articulación e integración intersectorial.

\section{LOS MODELOS CONCEPTUALES DE INTERPRETACIÓN PARA LA INTERVENCIÓN E IMPLEMENTACIÓN LOCAL}

La noción de Deuda Social Educativa expresa la existencia de grupos de niños, adolescentes y adultos con muy bajo nivel educativo o con educación de baja calidad; así la deuda social educativa significa un reto para la expansión de las ofertas escolares en vías de poder garantizar el acceso de toda la población excluida y la permanencia en el sistema.

La deuda social educativa en contextos de segmentación socioeducativa comprende problemas de accesibilidad, barreras y escenarios complejos. El acceso de la población al conjunto de bienes y servicios sociales depende de variables de orden cultural, físico y económico, mientras las barreras al acceso a esos bienes y servicios supone considerar las limitaciones del sistema oferente, el estado y el sector privado. Las medidas de políticas en educación y formación para el trabajo en las últimas décadas han configurado escenarios complejos por el resquebrajamiento del sistema educativo, la fragmentación de agentes e instituciones y la yuxtaposición de objetivos con la política social y de empleo.

La comprensión de las situaciones de segmentación y diferenciación sociales requiere un acercamiento a nivel de los ámbitos locales, desde la óptica de las políticas públicas a nivel nacional y provincial.

\footnotetext{
1 Proyecto "Educación y formación para el trabajo. Deuda social con jóvenes y adultos y mercado de ilusiones de corto plazo" (UBACyT Programación Científica 2011-2014) y Proyecto "Los escenarios complejos de la educación y formación para el trabajo: vigencia, nuevos sentidos y el papel de los actores sociales. (UBACyT Programación Científica 2014 -2017GC), Dirección: Graciela C. Riquelme, sede: Programa Educación, Economía y Trabajo (CONICET-PEET-IICE/UBA), página web: https://educacion-economiatrabajo-peet.org/.
} 
La interpretación de la atención de las demandas sociales por educación y formación para el trabajo de la población joven y adulta de 18 años y más, debe considerar una serie de las dimensiones y variables que se traducen centralmente en considerar:

a) la estructura social de la población y la deuda social con estos grupos originada en los déficits educativos de los jóvenes y adultos de 18 años y más, los problemas de accesibilidad a las instancias educativas;

b) los escenarios complejos de la educación y formación para el trabajo que diseñan o no alternativas de atención para superar estas barreras.

\section{Cuadro 1: Encuadre interpretativo de la atención de las demandas sociales por educación y formación para el trabajo}

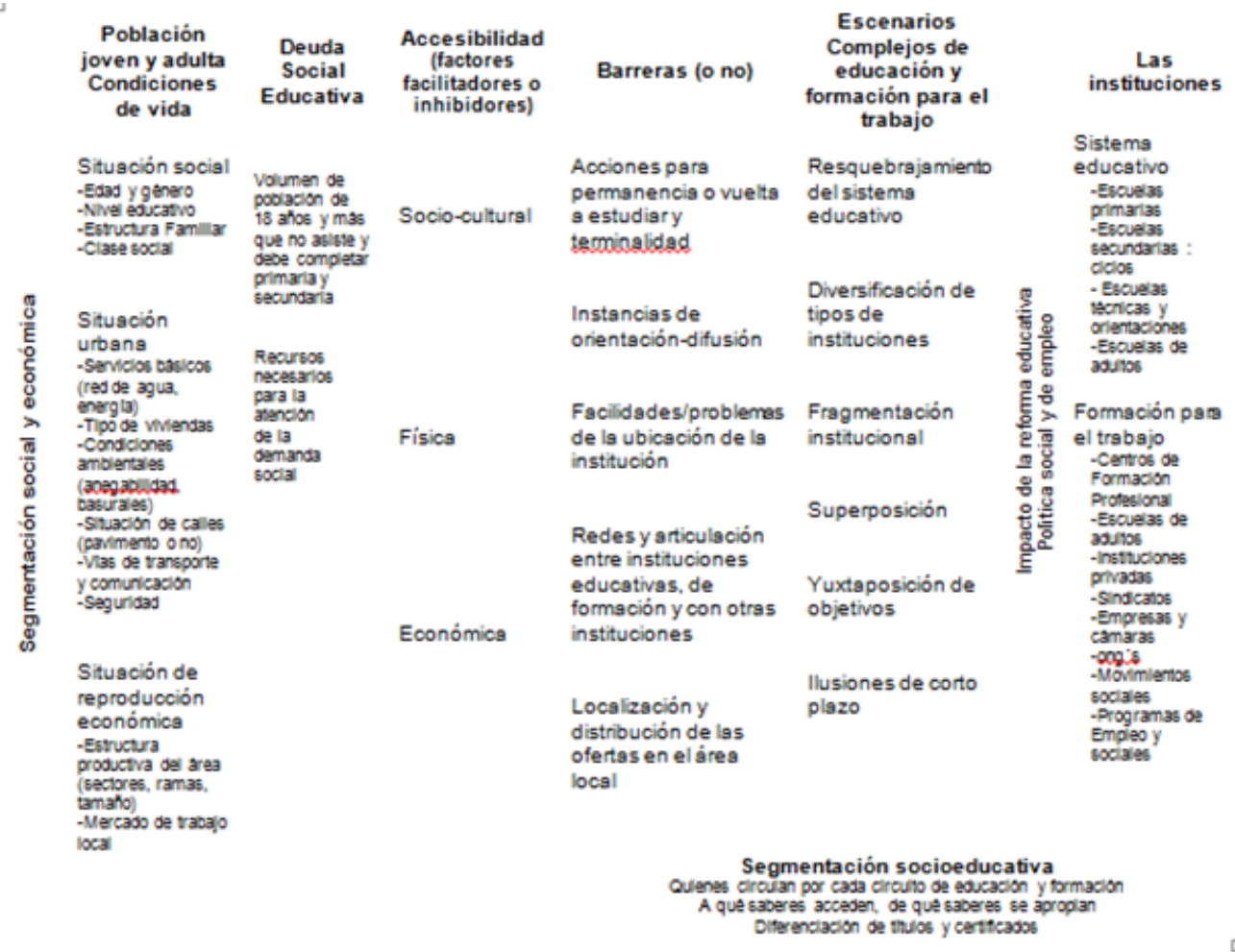

Fuente: Riquelme, G.C. (1978) y PEET-IICE/UBA Proyectos de Investigación UBACyT 2011 2014 y UBACyT 2014-2017 op cit. 
El encuadre conceptual nos sitúa en el desafío de articular los niveles macro, meso y micro en la comprensión de las políticas públicas de educación y formación para el trabajo y la configuración que adoptan en las áreas locales, en las instituciones y en las prácticas de los sujetos. Si bien las políticas públicas parecen definirse en las esferas nacional y provincial, es en los ámbitos locales en los que se concreta el interjuego entre las necesidades educativas de la población y las características del sistema de prestación de educación y formación y se definen la trama de oportunidades reales de acceso y permanencia de los sujetos de los diferentes grupos sociales.

\section{Cuadro 2. Re-construcción de conocimientos en ida y vuelta de lo meso a lo micro}
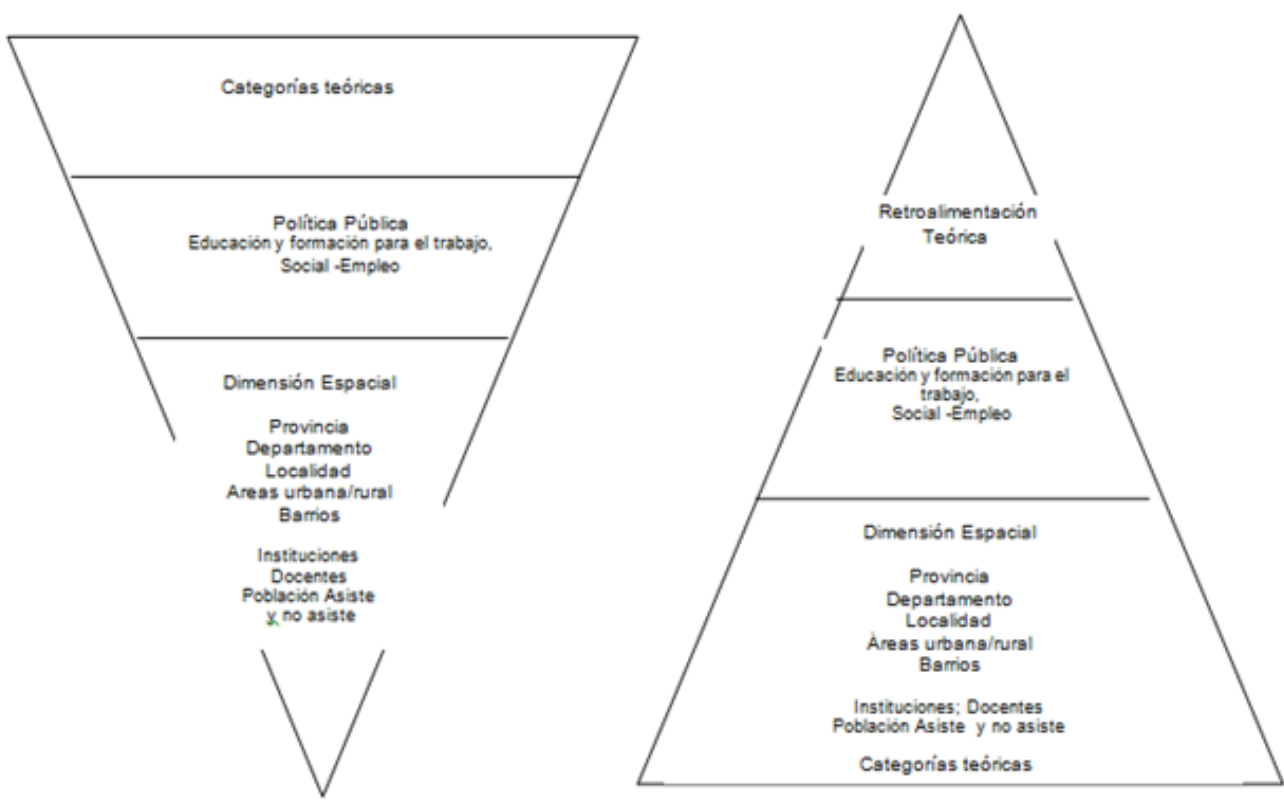

Fuente: PEET-IICE/UBA UBACyT 2011 - 2014 y UBACyT 2014-2017 op cit.

La interpretación de las condiciones que intervienen en el acceso y permanencia de la población joven y adulta al sistema de educación y formación para el trabajo en contextos de segmentación socioeducativa y diferenciación requiere una perspectiva que incluya diversas unidades de análisis:

- las políticas y programas dirigidos a la población joven y adulta, que se diseñan principalmente desde el gobierno nacional pero también desde los gobiernos provinciales en el sector educación, trabajo y desarrollo social, reconociendo las orientaciones, los objetivos y acciones que se implementan; 
- territorial, al tomar dos provincias con orientaciones políticas y educativas diferentes, como la provincia de Buenos Aires y Santa Fe, diferentes localidades del norte de la provincia de Buenos Aires (Campana y Zarate) y sur de Santa Fe (Villa Constitución-Empalme y Rosario), y, en el corto plazo, localidades del sur del conurbano bonaerense (Avellaneda, Berazategui, Florencio Varela) de manera a comparar el funcionamiento de las dimensiones y variables en contexto diferentes;

- institucional, referido a las características de las instituciones de educación y formación para el trabajo que atienden la demanda efectiva de los jóvenes y adultos, implementado y recontextualizando las políticas nacionales, provinciales y aún locales (Bernstein, 2001). En cada área urbana se han reconstruido los circuitos educativos, que vinculan el perfil institucional con el contexto socio económico; la dotación diferencial de recursos, la relación con los programas educativos, sociales y de empleo y la atención de las barreras al acceso.

- los propios sujetos, jóvenes y adultos con bajo nivel educativo, que asisten y no asisten a distintas ofertas educativas y deben enfrentar distintos tipos de las barreras de acuerdo a su situación socioeconómica y laboral.

\section{ACERCA DEL DERECHO A LA EDUCACIÓN Y DEUDA SOCIAL CON JÓVENES Y ADULTOS ${ }^{2}$}

El fracaso de las políticas modernizantes neoliberales que impulsaron programas de mercado en la sociedad latinoamericana, desde fines de los ochenta, contribuyó al reconocimiento de la educación como clave en la cohesión social e influyó para el cambio del discurso de la austeridad presupuestaria post-ajuste y la aceptación de la necesidad de invertir en educación por el reconocimiento de la existencia de grandes sectores de población excluida del sistema educativo.

La educación, en nuestra perspectiva, la entendemos como un derecho social, como garantía de igualdad de oportunidades para acceder a la educación primaria y secundaria y aún universitaria y no sólo como "educación para todos", si por ella se entiende nada más que educación primaria.

La noción de "Deuda Social Educativa" es definida en esta investigación como la cantidad de recursos necesarios para el cumplimiento de la finalización del nivel secundario de los niños, adolescentes y jóvenes que se encuentran excluidos del sistema, permite objetivar el problema en términos de aquello que el Estado 
-a través de todas sus jurisdicciones y con la participación de la sociedad- debe poder proyectar en términos de inversión, planificación y gestión educativa para saldar dicha deuda teniéndose como parámetro el derecho a una educación.

En referencia a este derecho adquirido de vivir en sociedad, también Lo Vuolo (2001) otorga otro matiz al concepto de deuda social. En este marco, las distintas políticas o acciones institucionales desde el Estado influyen sobre el sistema de "créditos sociales" para los ciudadanos, con el objeto de satisfacer sus necesidades en el mercado (necesitan poseer previamente dinero que lo acredite a tales fines y no son neutras). A través de ellas se definen diversas acciones que derivarán luego en situaciones de inclusión/exclusión social; de manera que, todos los individuos pertenecientes a un Estado, al pagar impuestos, otorgan un "crédito original" sobre el Estado para que éste, luego, cumpla con sus obligaciones de reutilización de aquellos tributos. Así el concepto de "deuda social" nacería, en esta perspectiva, como un no cumplimento por parte del Estado de aquellas obligaciones y posee un doble significado.

Es, por un lado, una "deuda" del Estado hacia la Sociedad Civil en tanto este no haya sido cancelado correctamente el "crédito original" que aquella le ha otorgado bajo la forma de impuestos; por otro lado la sociedad, ante la merma en sus "créditos sociales" está imposibilitada o posee una menor capacidad de reclamar dinero y, por lo tanto, de hacer frente a las deudas necesarias que resultan del sistema de pagos. En otras palabras, la propia existencia del fenómeno de exclusión de los individuos del sistema de pagos recíproco representa la forma en que se expresa una determinada "deuda social" del Estado para con esos ciudadanos.

La perspectiva del uso de un modelo se basa en el reconocimiento, en primer término, de la multidimensionalidad de la problemática a encarar y, en segunda instancia y derivado del anterior diagnóstico, de la situación por la cual, para la atención de la Deuda Social Educativa (DSE) se requiere una coordinación institucional desde las grandes líneas de programación de la política social hasta su traducción en objetivos y metas específicas a atender.

En este caso, el modelo representa un ejercicio intelectual que busca poner de manifiesto las distintas dimensiones implicadas en la atención de la Deuda Social Educativa, (que coinciden con el Encuadre interpretativo de la atención de las demandas sociales por educación y formación para el trabajo) y sobre las cuales sería importante intervenir a través de acciones planificadas de la educación o de determinadas acciones puntuales de atención.

El diseño señala las tres dimensiones para la caracterización de la oferta y la demanda social en educación: 
1. la diferenciación social y económica de la población;

2. la segmentación institucional tanto educativa como de los programas sociales, que implica la existencia de tipos y calidades de prestaciones escolares de acuerdo a grupos sociales;

3. la diferenciación espacial en la dotación de recursos del sistema educativo y de la formación para el trabajo, es decir, la desagregación de la DSE por provincia y ámbitos locales.

Esta investigación ha avanzado en las indagaciones de estas dimensiones a nivel local, pero debe enfrentar serias dificultades de falta de información para evaluar la articulación entre las políticas y medidas nacionales, provinciales y municipales, además de la evaluación de los impactos de las asignaciones condicionadas.

\section{UNA APROXIMACIÓN A LA DEUDA SOCIAL EDUCATIVA CON LA POBLACIÓN JOVEN Y ADULTA}

En 2009, las discusiones/en reuniones de trabajo ${ }^{3}$ acerca del diseño de un modelo de atención, se logró revisar críticamente este ejercicio. Así se planteó que "el máximo maximorum consistiría en definir la Deuda Social Educativa (DSE) como la distancia histórica o proyectada y una situación (super) ideal en la que todos los habitantes hubieran alcanzado (o estuvieran en el proceso de hacerlo según a edad) el nivel terciario completo, todo ello en condiciones razonables de calidad y eficiencia de la educación" (Riquelme y Monza, 2009, et al, citado en Riquelme, 2013; p. 85).

Sin duda, la realidad impone límites políticos, burocráticos a los derechos y su efectiva concreción, por eso luego reconocíamos en el debate que "el núcleo duro de la DSE sería el criterio básico de la obligatoriedad legal en el tramo etario central, entre 5 y 24 años, como expectativa de que las políticas públicas atendieran y garantizaran el cumplimiento de la obligatoriedad para la población infantil y adolescente" (Riquelme, 2013; p. 85).

La población, prioridad definida en los proyectos previos, y que coinciden con las orientaciones de las políticas del Estado, para estimar los recursos necesarios del gasto social, es sin duda:

- la población infantil y adolescente de 5 a 18 años, entendida como en el tramo restringido de la obligatoriedad legal;

3 Riquelme, G. C. y Kodric, A. (2011); Riquelme, G. C., Costa, A. y Kodric, A. (2011); Riquelme, G. C. y Monza, A., con la colaboración de Herger, N. y Kodric, A. (2009); Riquelme, Graciela C. (2007). 
- la población infantil de 3 y 4 años, pues constituye el periodo óptimo de estimulación básica para el desarrollo educativo.

El actual proyecto avanza sobre el derecho de los jóvenes y adultos que no han concluido satisfactoriamente los ciclos educativos obligatorios (educación básica y secundaria) ni a la formación terciaria y universitaria.

El volumen de población de 18 años y más que no asiste y debe completar el nivel primario y secundario, requeriría considerar y estimar la presión poblacional según las siguientes dimensiones:

- las características socio demográficas, de origen social y de ocupación de la población con bajo nivel educativo;

- la localización espacial, entendida como el acceso y permanencia diferencial de la población en áreas locales;

- los escenarios de la educación y formación para el trabajo y la oferta de las instituciones configurados en función de la segmentación en la dotación de recursos de las escuelas y la existencia de circuitos diferenciales de acuerdo a los grupos sociales;

- los recursos para responder a la deuda social educativa, como ejercicio de estimación que da cuenta de los montos necesarios en relación con los gastos del sector público en educación y en algunos programas sociales y de empleo.

Cuadro 3. Características sociales y económicas de Argentina, provincias y áreas seleccionadas. 2010.

\begin{tabular}{|c|c|c|c|c|c|c|}
\hline \multirow[b]{2}{*}{$\begin{array}{c}\text { Total país, } \\
\text { provincias } \\
\text { y áreas } \\
\text { seleccionadas }\end{array}$} & \multicolumn{2}{|c|}{ Hogar y Vivienda } & \multicolumn{4}{|c|}{ Población } \\
\hline & $\begin{array}{l}\text { \%Hogares } \\
\text { con NBI }\end{array}$ & $\begin{array}{c}\% \text { de } \\
\text { Viviendas } \\
\text { con calidad } \\
\text { insuficiente } \\
\text { de las } \\
\text { conexiones } \\
\text { a servicios } \\
\text { básicos }\end{array}$ & $\begin{array}{l}\text { \% Población } \\
\text { de } 10 \text { y más } \\
\text { analfabetismo }\end{array}$ & $\begin{array}{c}\% \text { PEA ( } 15 \text { a } \\
64 \text { años) }\end{array}$ & $\begin{array}{c}\% \text { PEA } \\
\text { desocupada } \\
\text { (15 a } 64 \\
\text { años) }\end{array}$ & $\begin{array}{c}\% \\
\text { Población } \\
\text { de } 12 \text { a } \\
18 \text { años } \\
\text { ocupada }\end{array}$ \\
\hline Total País & 9,1 & 30,3 & 1,9 & 73,1 & 6,1 & 26,4 \\
\hline $\begin{array}{c}\text { Provincia } \\
\text { Buenos Aires }\end{array}$ & 8,1 & 39,0 & 1,4 & 76,3 & 6,2 & 29,8 \\
\hline Campana & 8,7 & 38,9 & 1,2 & 74,8 & 4,8 & 28,0 \\
\hline Zárate & 9,1 & 29,9 & 1,4 & 72,9 & 5,9 & 26,2 \\
\hline $\begin{array}{c}\text { Provincia Santa } \\
\text { Fe }\end{array}$ & 6,4 & 35,8 & 1,8 & 73,4 & 6,1 & 26,4 \\
\hline Rosario & 6,0 & 23,8 & 1,6 & 75,8 & 6,6 & 26,7 \\
\hline
\end{tabular}

Fuente: Proyecto UBACyT 2011-2014 y PIP 2012-2014 sobre la base de INDEC Censo Nacional de Población, Hogares y Viviendas 2010. CEPAL/CELADE Redatam+SP 
Así entendida la atención de la deuda social educativa podría contribuir con la planificación educativa en tanto permite identificar la demanda social por educación, es decir, la población excluida del sistema educativo formal antes de haber completado la secundaria, con el objeto de diseñar diversas instancias para la inclusión educativa. El sombreado destaca la población prioritaria correspondiente a los más jóvenes y el primer segmento de los adultos 18-24 y 25-29.

\section{Cuadro 4. Perfil educativo de la población de 18 años y más. Provincia de Buenos Aires y Santa Fe. 2010. En porcentajes.}

\begin{tabular}{|c|c|c|c|c|c|c|c|c|c|}
\hline \multirow{2}{*}{ Provincia 5} & \multirow{2}{*}{ Total } & \multirow{2}{*}{$\begin{array}{c}\text { Nunca } \\
\text { asistió y } \\
\text { primario } \\
\text { inc om pleto }\end{array}$} & \multirow{2}{*}{$\begin{array}{l}\text { Primario } \\
\text { com pleto }\end{array}$} & \multicolumn{2}{|c|}{ Secundario } & \multicolumn{2}{|c|}{ Supe rior no unive rsitario } & \multicolumn{2}{|c|}{ Univers ita rio } \\
\hline & & & & Inc om pleto & Comple to & Inc om ple to & Completo & Incomple to & Comple to \\
\hline Bue nos $A$ ires & 100,0 & 12,6 & 27,3 & 18,2 & 20,6 & 3,3 & 5,2 & 7,1 & 5,6 \\
\hline 18 a 24 años & 100,0 & 7,3 & 9,6 & 36,8 & 20,9 & 6,4 & 1,4 & 16,3 & 0,9 \\
\hline 25 a 29 años & 100,0 & 6,1 & 15,6 & 20,2 & 30,0 & 5,3 & 5,4 & 11,5 & 5,7 \\
\hline 30 a 39 años & 100,0 & 7,0 & 25,5 & 17,6 & 25,2 & 2,9 & 7,8 & 5,5 & 8,5 \\
\hline 40 años ymás & 100,0 & 17,5 & 35,6 & 12,4 & 16,9 & 2,1 & 5,4 & 4,0 & 6,0 \\
\hline Santa Fe & 100,0 & 13,3 & 27,0 & 15,2 & 20,5 & 3,8 & 8,4 & 7,5 & 6,4 \\
\hline 18 a 24 años & 100,0 & 7,0 & 11,7 & 32,8 & 18,3 & 8,0 & 2,0 & 18,8 & 1,1 \\
\hline 25 a 29 años & 100,0 & 6,1 & 17,9 & 18,9 & 26,0 & 5,9 & 7,2 & 12,3 & 7,4 \\
\hline 30 a 39 aก̂́os & 100,0 & 8,0 & 23,8 & 14,1 & 25,0 & 3,4 & 10,4 & 5,4 & 9,9 \\
\hline 40 años ymás & 100,0 & 18,7 & 34,7 & 9,9 & 18,3 & 1,8 & 6,2 & 3,8 & 6,8 \\
\hline
\end{tabular}

Fuente: PEET-IICE/UBA UBACyT 2011 - 2014 y UBACyT 2014-2017 sobre la base del Censo 2010.

Los desafíos para la educación primaria y secundaria de jóvenes y adultos se expresan en las estimaciones de la población mayor de 18 años que no terminó la primaria ni la secundaria, y que suponen una demanda social educativa potencial para los sistemas educativos provinciales. 
Cuadro 5. Tasa de escolarización bruta por nivel. Población de 18 años y más con hasta secundario incompleto. Provincia de Buenos Aires y Santa Fe. 2010.

\begin{tabular}{|l|c|c|}
\hline \multirow{2}{*}{} & \multicolumn{2}{|c|}{ Provincia } \\
\cline { 2 - 3 } & Buenos Aires & Santa Fe \\
\hline Matricula Nivel Primario Adultos (1) & 98.027 & 18.324 \\
\hline Población 18 y más con hasta primario incompleto (2) & 1.365 .524 & 303.269 \\
18-24 años & $(127.218)$ & $(25.713)$ \\
25-29 años & $(73.146)$ & $(15.680)$ \\
\hline Tasa de escolarización bruta en primario de jóvenes y adultos (1) / (2) & 7,2 & 6,0 \\
\hline Matricula Nivel Secundario Adultos (3) & 182.095 & 25.636 \\
Población 18 y más con primaria completa o secundario incompleto (4) & 4.933 .131 & 959.485 \\
18-24 años & $(805.913)$ & $(163.331)$ \\
25-29 años & $(427.618)$ & $(89.137)$ \\
\hline Tasa de escolarización bruta en secundario de jóvenes y adultos (3) / (4) & 3,7 & 2,7 \\
\hline
\end{tabular}

Fuente: Proyecto UBACyT 2011-2014 y PIP 2012-2014 sobre la base de: a) Censo de Población 2010, INDEC; b) Relevamiento Anual 2014. DiNIECE.

Las tasas de escolarización son muy bajas, pues refieren a toda la población adulta - alrededor del $10 \%$ primaria de adultos y entre un 19 al 20\% secundaria adultos-, pero si consideramos la asistencia por edad, estas tasas serían algo más altas.

\section{UN EJERCICIO DE APLICACIÓN DE ESTIMACIÓN DE RECURSOS NECESARIOS PARA DE LA DEUDA SOCIAL EDUCATIVA ARGENTINA}

Sobre la base de una metodología especialmente elaborada para la investigación, la Deuda Social Educativa puede entenderse como los recursos necesarios para atender a las necesidades socioeducativas de las personas excluidas del sistema educativo, en este caso los jóvenes y adultos mayores de 18 años analfabetos o que no han terminado la primaria y la secundaria. Pese a que la DSE abarcaría a toda la estructura etaria de la población, a los fines de este trabajo, tal como se planteó antes, se prioriza la atención del grupo más joven de 18 a 29 años, y que no están asistiendo al sistema escolar.

El ejercicio se realiza para el año 2010 considerando la estimación de recursos necesarios para la atención de la Deuda Social Educativa a partir de cálculos para escenarios alternativos tomando como referencia los siguientes datos de base: el gasto por alumno en el sector estatal para el año 2009 obtenido de las cifras oficiales del sector educación, el gasto público consolidado en educación y el gasto público social. 
Estos datos para las fechas de la construcción de los escenarios, son los que permiten luego inferir las partidas adicionales o incrementales sobre los presupuestos de educación que se deberían aplicar a la expansión de la atención en acuerdo con los parámetros definidos para cada escenario de atención.

La educación y formación para el trabajo tendría como desafío garantizar el derecho retroactivo sobre esta población a la obligatoriedad de la primaria y secundaria, más aún considerando las características excluyente y discriminatoria de vastos sectores sociales del sistema educativo en décadas pasadas.

A continuación se resumen distintos cálculos basados en hipótesis alternativas, con el propósito de contar con una referencia respecto a los recursos adicionales involucrados en el objetivo de atender la deuda social educativa con la población de 18 y más de las provincias de Buenos Aires y Santa Fe.

Cuadro 6. Deuda social educativa con la población de 18 a 40 años que nunca asistió y que asistió hasta secundaria incompleta. Resumen de las algunas hipótesis preliminares para cada uno de los escenarios. Provincia de Buenos Aires y Santa Fe.

\begin{tabular}{|c|c|c|c|c|}
\hline & Hipótesis 1 & Hipótesis 2 & Hipótesis 3 & Hipótesis 4 \\
\hline $\begin{array}{l}\text { Gasto Promedio } \\
\text { por Alumno }\end{array}$ & $\begin{array}{c}\text { Gasto por alumno } \\
\text { del sector estatal } \\
\text { correspondiente } \\
\text { a: Buenos Aires } \\
\text { (2009), Santa Fe } \\
\text { (2010). }\end{array}$ & $\begin{array}{c}\text { Gasto por alumno } \\
\text { del sector estatal } \\
\text { correspondiente } \\
\text { a: Buenos Aires } \\
(2009), \text { Santa Fe } \\
\text { (2010). }\end{array}$ & $\begin{array}{c}\text { Gasto por alumno } \\
\text { del sector estatal } \\
\text { correspondiente } \\
\text { a: Buenos Aires } \\
(2009), \text { Santa Fe } \\
\text { (2010). }\end{array}$ & $\begin{array}{c}\text { Gasto por alumno } \\
\text { del sector estatal } \\
\text { correspondiente } \\
\text { a: Buenos Aires } \\
\text { (2009), Santa Fe } \\
\text { (2010). }\end{array}$ \\
\hline $\begin{array}{l}\text { Atención del } 100 \% \\
\text { de la DSE }\end{array}$ & $\begin{array}{c}\text { Atención inmediata } \\
\text { de la población que } \\
\text { nunca asistió (18-40 } \\
\text { años) }\end{array}$ & $\begin{array}{c}\text { Hipótesis } 2.1 \\
\text { Atención inmediata } \\
\text { de la población } \\
\text { que asistió con } \\
\text { secundario } \\
\text { incompleto (18-24 } \\
\text { años). } \\
\text { Hipótesis } 2.2 \\
\text { Atención del 50\% } \\
\text { de la población } \\
\text { que asistió con } \\
\text { secundario } \\
\text { incompleto (18-24 } \\
\text { años) }\end{array}$ & $\begin{array}{l}\text { Atención inmediata } \\
\text { de la población } \\
\text { que asistió con } \\
\text { secundario } \\
\text { incompleto (18-24 } \\
\text { años) y, } \\
\text { Atención del } 50 \% \\
\text { de la población } \\
\text { que asistió con } \\
\text { secundario } \\
\text { incompleto (25-29 } \\
\text { años) }\end{array}$ & $\begin{array}{l}\text { Atención inmediata } \\
\text { de la población } \\
\text { que asistió con } \\
\text { secundario } \\
\text { incompleto (18-24 } \\
\text { años) y, } \\
\text { Atención del 50\% } \\
\text { de la población } \\
\text { que asistió con } \\
\text { secundario } \\
\text { incompleto (25-29 } \\
\text { años) y, } \\
\text { Atención del 30\% } \\
\text { de la población } \\
\text { que asistió con } \\
\text { secundario } \\
\text { incompleto (30-39 } \\
\text { años) }\end{array}$ \\
\hline $\begin{array}{l}\text { Diferenciación } \\
\text { en el gasto por } \\
\text { alumno según } \\
\text { nivel/edad escolar }\end{array}$ & No por el momento & No por el momento & No por el momento & No por el momento \\
\hline $\begin{array}{l}\text { Hipótesis de } \\
\text { mejora }\end{array}$ & No por el momento & No por el momento & No por el momento & No por el momento \\
\hline
\end{tabular}

Fuente: Elaboración propia sobre la base Proyectos UBACyT y PIP CONICET Sede: PEET-IICEFFyL/UBA 


\section{Cuadro 7. Hipótesis 1. Atención de la población que nunca asistió (18-40 años). En pesos corrientes y porcentaje incremental del gasto público educativo (GPE) provincial.}

\begin{tabular}{|c|c|c|c|c|}
\hline & $\begin{array}{c}\text { Demanda Social } \\
\text { Educativa (2010) }\end{array}$ & $\begin{array}{c}\text { Gasto por alumno } \\
\text { por alumno sector } \\
\text { estatal (2009) }\end{array}$ & $\begin{array}{c}\text { Deuda Social } \\
\text { Educativa (2010) }\end{array}$ & $\begin{array}{c}\text { \% incremental GPE } \\
\text { provincial (2009) }\end{array}$ \\
\hline Buenos Aires & 36.496 & 5.970 & 217.892 .069 & $1,07 \%$ \\
\hline Campana & 171 & 5.970 & 1.019 .429 & \\
\hline Zarate & 189 & 5.970 & 1.128 .387 & $\ldots$ \\
\hline Santa Fe & 8.625 & 7.740 & 66.752 .760 & $1,42 \%$ \\
\hline Rosario & 2.403 & 7.740 & 18.598 .259 & $\ldots$ \\
\hline
\end{tabular}

* El gasto por alumno en educación del sector estatal para Santa Fe y Rosario corresponde al año 2010 .

Fuente: Elaboración propia sobre la base Proyectos UBACy'T y PIP CONICET Sede: PEET-IICEFFyL/UBA

\section{Cuadro 8. Hipótesis 2.}

- 2.1. Atención de la totalidad de la población que asistió hasta secundario incompleto de 18-24 años. En pesos corrientes y porcentaje incremental del gasto público educativo (GPE) provincial.

- 2.2. Atención del 50\% del total de la población que asistió hasta secundario incompleto de 18-24 años. En pesos corrientes y porcentaje incremental del gasto público educativo (GPE) provincial.

\begin{tabular}{|c|c|c|c|c|c|c|c|}
\hline & \multicolumn{2}{|c|}{$\begin{array}{l}\text { Demanda Social } \\
\text { Educativa (2010) }\end{array}$} & \multirow{2}{*}{$\begin{array}{l}\text { Gasto por } \\
\text { alumno por } \\
\text { alumno } \\
\text { sector } \\
\text { estatal } \\
(2009)^{*}\end{array}$} & \multirow{2}{*}{$\begin{array}{c}\begin{array}{c}\text { Deuda Social } \\
\text { Educativa } \\
(2010)\end{array} \\
\text { Hipótesis } 2.1\end{array}$} & \multirow[b]{2}{*}{ Hipótesis 2.2} & \multicolumn{2}{|c|}{$\begin{array}{l}\text { \% incremental GPE } \\
\text { provincial (2009) }\end{array}$} \\
\hline & $\begin{array}{c}\text { Hipótesis } \\
\text { 2.1: } \\
\text { Atención } \\
\text { del } 100 \% \\
\text { de la } \\
\text { Deuda }\end{array}$ & $\begin{array}{c}\text { Hipótesis } \\
2.2: \\
\text { Atención } \\
\text { del } 50 \% \text { de } \\
\text { la Deuda }\end{array}$ & & & & $\begin{array}{c}\text { Hipótesis } \\
2.1\end{array}$ & $\begin{array}{c}\text { Hipótesis } \\
2.2\end{array}$ \\
\hline $\begin{array}{c}\text { Buenos } \\
\text { Aires }\end{array}$ & 877.034 & 438.517 & 5.970 & 5.236 .154 .896 & 2.618.077.448 & $25,81 \%$ & $12,90 \%$ \\
\hline Campana & 11.024 & 5.512 & 5.970 & 65.815 .095 & 32.907 .547 & $\ldots$ & $\ldots$ \\
\hline Zarate & 13.392 & 6.696 & 5.970 & 79.951 .272 & 39.975 .636 & $\ldots$ & $\ldots$ \\
\hline Santa Fe & 131.912 & 65.956 & 7.740 & 1.020.949.211 & 510.474 .606 & $21,78 \%$ & $10,89 \%$ \\
\hline Rosario & 148.030 & 74.015 & 7.740 & 1.145 .691 .053 & 572.845 .527 & $\ldots$ & $\ldots$ \\
\hline
\end{tabular}

Fuente: Elaboración propia sobre la base Proyectos UBACyT y PIP CONICET Sede: PEET-IICEFFyL/UBA 


\section{Cuadro 9. Hipótesis 3.}

- Atención de la totalidad de la población que asistió hasta secundario incompleto de 1824 años. En pesos corrientes y porcentaje incremental del gasto público educativo (GPE) provincial.

- Atención del 50\% del total de la población que asistió hasta secundario incompleto de 25-29 años. En pesos corrientes y porcentaje incremental del gasto público educativo (GPE) provincial.

\begin{tabular}{|c|c|c|c|c|c|c|c|c|c|c|}
\hline & \multicolumn{3}{|c|}{$\begin{array}{l}\text { Demanda Social Educativa } \\
\qquad(2010)\end{array}$} & \multirow{2}{*}{$\begin{array}{c}\text { Gasto } \\
\text { por } \\
\text { alumno } \\
\text { por } \\
\text { alumno } \\
\text { sector } \\
\text { estatal } \\
(2009)^{*}\end{array}$} & \multicolumn{3}{|c|}{ Deuda Social Educativa (2010) } & \multicolumn{3}{|c|}{$\begin{array}{l}\% \text { incremental GPE } \\
\text { provincial }(2009)\end{array}$} \\
\hline & Total & $18-24$ & $25-29$ & & Total & $18-24$ & $25-29$ & Total & $18-24$ & $25-29$ \\
\hline $\begin{array}{l}\text { Buenos } \\
\text { Aires }\end{array}$ & 1.100 .944 & 877.034 & 223.911 & 5.970 & 6.572 .967 .754 & 5.236 .154 .896 & 1.336 .812 .858 & $32,40 \%$ & $25,81 \%$ & $6,59 \%$ \\
\hline Campana & 15.636 & 11.024 & 4.613 & 5.970 & 93.353 .103 & 65.815 .095 & 27.538 .009 & $\ldots$ & $\ldots$ & $\ldots$ \\
\hline Zarate & 18.748 & 13.392 & 5.356 & 5.970 & 111.928.199 & 79.951 .272 & 31.976 .927 & $\ldots$ & $\ldots$ & $\ldots$ \\
\hline Santa Fe & 179.387 & 131.912 & 47.475 & 7.740 & 1.388 .386 .721 & 1.020 .949 .211 & 367.437 .510 & $29,61 \%$ & $21,78 \%$ & $7,84 \%$ \\
\hline Rosario & 209.012 & 148.030 & 60.983 & 7.740 & 1.617.671.210 & 1.145 .691 .053 & 471.980 .157 & .. & $\ldots$ & $\cdots$ \\
\hline
\end{tabular}

Fuente: Elaboración propia sobre la base Proyectos UBACyT y PIP CONICET Sede: PEET-IICEFFyL/UBA

\section{Cuadro 10. Hipótesis 4.}

- Atención de la totalidad de la población que asistió hasta secundario incompleto de 1824 años. En pesos corrientes y porcentaje incremental del gasto público educativo (GPE) provincial.

- Atención del 50\% del total de la población que asistió hasta secundario incompleto de 25-29 años. En pesos corrientes y porcentaje incremental del gasto público educativo (GPE) provincial.

- Atención del 30\% del total de la población que asistió hasta secundario incompleto de 30-39 años. En pesos corrientes y porcentaje incremental del gasto público educativo (GPE) provincial.

\begin{tabular}{|c|c|c|c|c|c|c|c|c|c|c|}
\hline & \multicolumn{4}{|c|}{ Demanda Social Educativa (2010) } & \multirow{2}{*}{$\begin{array}{l}\text { Gasto por } \\
\text { alumno } \\
\text { por } \\
\text { alumno } \\
\text { sector } \\
\text { estatal } \\
(2009)^{*}\end{array}$} & \multicolumn{4}{|c|}{ Deuda Social Educativa (2010) } & \multirow{2}{*}{$\begin{array}{c}\% \\
\text { incremental } \\
\text { GPE } \\
\text { provincial } \\
(2009)\end{array}$} \\
\hline & Total & $18-24$ & $25-29$ & $30-39$ & & Total & $18-24$ & $25-29$ & $30-39$ & \\
\hline $\begin{array}{c}\text { Buenos } \\
\text { Aires }\end{array}$ & 1.408 .222 & 877.034 & 223.911 & 307.277 & 5.970 & 8.407 .506 .016 & 5.236 .154 .896 & 1.336 .812 .858 & 1.834 .538 .261 & $41,44 \%$ \\
\hline Campana & 21.077 & 11.024 & 4.613 & 5.441 & 5.970 & 125.838 .103 & 65.815 .095 & 27.538 .009 & 32.484 .999 & $\ldots$ \\
\hline Zarate & 25.158 & 13.392 & 5.356 & 6.410 & 5.970 & 150.200 .210 & 79.951 .272 & 31.976 .927 & 38.272 .011 & $\ldots$ \\
\hline Santa Fe & 236.009 & 131.912 & 47.475 & 56.622 & 7.740 & 1.826 .616 .030 & 1.020 .949 .211 & 367.437 .510 & 438.229 .309 & $38,96 \%$ \\
\hline Rosario & 274.731 & 148.030 & 60.983 & 65.719 & 7.740 & 2.126 .306 .887 & 1.145 .691 .053 & 471.980 .157 & 508.635 .677 & $\ldots$ \\
\hline
\end{tabular}

Fuente: Elaboración propia sobre la base Proyectos UBACyT y PIP CONICET Sede: PEET-IICE-

FFyL/UBA 
El cálculo para la estimación de los recursos incrementales de los presupuestos de las provincias de la investigación - Buenos Aires y Santa Fe- y las áreas locales consideró (1) la definición de la población joven y adulta hasta 40 años que nunca asistió y excluida del sistema con hasta secundaria incompleta; (2) el gasto por alumno del sector estatal como proxy del costo por alumno y (3) los gastos necesarios incrementales por la multiplicación de (1) y (2); y el (4) a partir de este dato el porcentaje de recursos incrementales sobre el presupuesto o gasto público educativo provincial (GPE).

La estimación de recursos incrementales de los presupuestos para atender a las necesidades educativas de la población excluida del sistema fue simplificada en este ejercicio, pero constituye un aporte conceptual y metodológico para la implementación de las leyes de financiamiento educativo y la planificación en términos del monitoreo y garantía del el acceso y permanencia a los niveles primario y secundario de la población en edad de obligatoriedad; y más aún para los adolescentes, jóvenes y adultos de bajo nivel educativo en el marco de la educación y formación a lo largo de la vida.

\section{NOTAS FINALES}

El presente artículo a partir de la noción de derecho a la educación sostiene la existencia de una deuda social educativa, desde una perspectiva de la política y planificación de la educación de jóvenes y adultos. En tal sentido, se ha argumentado que el reconocimiento y cumplimiento de dicho derecho debería ser retroactivo para la mejora de las condiciones de sociabilidad de todos los ciudadanos, y no debería restringirse o acotarse su alcance a la finalización de un determinado nivel educativo o determinado grupo etario de la población.

A su vez, la no realización y cumplimiento del derecho a la educación la hemos definido como una Deuda Social Educativa (DSE) del Estado para con sus ciudadanos. Esta deuda se expresaría tanto bajo la forma de exclusión de una determinada porción de la población del sistema educativo, como bajo la forma de una merma cualitativa respecto a la calidad de la educación brindada. En tanto, la educación (como otras tantas prestaciones brindadas por el Estado) representa para sus ciudadanos una suerte de "créditos sociales" que mejoran sus condiciones de demanda en el sistema recíproco de pagos y, también, sus condiciones de demanda respecto a reclamos sucesivos por la ampliación y el mejoramiento de nuevos derechos.

Este artículo presentó avances de una investigación cuyo objetivo general es contribuir a la comprensión de la complejidad, diversificación y superposición de ofertas de educación y formación para el trabajo y evaluar la dimensión de la deuda social educativa en ella involucrada y la estimación de los recursos 
individuales y sociales aplicados y no apropiados por diferentes ámbitos de la política educativa y social (trabajo, desarrollo social y salud).

La investigación cuenta con resultados acerca de las evidencias del impacto de las políticas públicas en los ámbitos locales y las instituciones de educación y formación para el trabajo ${ }^{4}$. El encuadre conceptual, al articular los niveles macro, meso y micro permite una mayor comprensión de la implementación de las políticas públicas y la configuración que adoptan en las áreas locales. Junto a ello, el abordaje cuanti-cualitativo nos permite identificar factores que intervienen en la regulación de las políticas a nivel del Estado nacional, provincial, regional y local en términos de los problemas de coordinación e integración intersectorial.

\section{BIBLIOGRAFÍA}

Bélanger, P. y Federighi, P (2004) Análisis transnacional de las políticas de educación y formación de adultos. La difícil liberación de las fuerzas productivas. Colección Ideas en debate. Miño y Dávila Editores. Buenos Aires.

Bernstein, B. (2001). La estructura del discurso pedagógico. Clases, códigos y control (Volumen VI), Madrid, Fundación Paideia, Morata.

Fitoussi, J. P. y Rosanvallon, P. (2003). La nueva era de las desigualdades, Ediciones Manantial, Buenos Aires.

Lo Vuolo, R. (2001) Alternativas. La economía como cuestión social, Ediciones Altamira, Buenos Aires.

Riquelme, G. C. (2013) “La Deuda Social Educativa y el Derecho a la Educación: la importancia de los ejercicios de estimación de recursos para la planificación educativa", en Riquelme, G. C. y A. Kodric (2013) Deuda Social Educativa. Atención del derecho a la Educación. Ediciones Lumiere SA. Buenos Aires.

Riquelme, G. C. y A. Kodric (2013) Deuda Social Educativa. Atención del derecho a la Educación.Ediciones Lumiere SA. Buenos Aires.

Riquelme, G. C., Costa, A. y Kodric, A. (2011) Escenarios alternativos de estimación de recursos del gasto social en un modelo de atención de la deuda social educativa con la población infantil y adolescente de Argentina.

4 En la página web del Programa Educación, Economía y Trabajo (PEET-IICE/UBA) pueden consultarse publicaciones que dan cuenta de estos resultados: https://educacion-economia-trabajo-peet

52 - RBPAE - v. 33, n. 1, p. 037 - 054, jan./abr. 2017 
Serie de Cuadernos del Educación, Economía y Trabajo, no 27. CONICET. Programa Educación, Economía y Trabajo (PEET), IICE, FFyL, UBA. Buenos Aires. Julio.

Riquelme, G. C. y Monza, A., con la colaboración de Herger, N. y Kodric, A. (2009) Hacia el diseño de modelos de atención del derecho a la educación: antecedentes, informes de base y relatoría de reuniones de trabajo". Serie de Cuadernos del Educación, Economía y Trabajo, nº 24. CONICET. Programa Educación, Economía y Trabajo (PEET), Instituto de Investigaciones en Ciencias de la Educación (IICE), Facultad de Filosofía y Letras (FFyL). UBA. Buenos Aires. Febrero-junio.

Riquelme, G. C. (2007) La deuda social educativa en Argentina: práctica y cumplimiento del derecho a la educación. Serie de Cuadernos del Educación, Economía y Trabajo, no 16. CONICET. Programa Educación, Economía y Trabajo (PEET), Instituto de Investigaciones en Ciencias de la Educación (IICE), Facultad de Filosofía y Letras (FFyL). UBA. Buenos Aires.

Riquelme, G. C. y Herger, N. (2005) "La explosión y fragmentación de la educación y formación para el trabajo en Argentina: resignificación y desafíos en la perspectiva de los jóvenes y adultos", en Archivos Analíticos de Políticas Educativas, volumen 13, número 39, septiembre 26, 2005. Editor: Sherman Dorn, College of Education. University of South Florida. http://epaa.asu.edu/ epaa/v13n39.

Riquelme, G. C. (2004). La educación secundaria antes y después de la reforma: efectos distributivos del gasto público, Buenos Aires, Facultad de Filosofía y Letras-UBA, Miño y Dávila.

Riquelme, G. C. (1978) Estudio Metodológico sobre la integración de la dinámica poblacional en la planificación de la educación para el desarrollo de zonas rurales, UNESCO. Oficina Regional de Educación para América Latina y el Caribe (OREALC). Fundación para el desarrollo de América Latina (FUDAL).

Steinberg, C; Cetrángolo, O y Gatto, F (2011). Desigualdades territoriales en la Argentina. Insumos para el planeamiento estratégico del sector educativo. Comisión Económica para América Latina y Caribe (CEPAL). Documento de Proyecto. Santiago de Chile, Chile. Febrero. 
GRACIELA CLOTILDE RIQUELME: Investigadora Principal del CONICET. Doctora de la Universidad de Buenos Aires (UBA), Directora del Programa Educación, Economía y Trabajo del Instituto de Investigaciones en Ciencias de la Educación (IICE-FFyL/UBA). Profesora Titular Ordinaria de Economía de la Educación (UBA). Investigadora Visitante School of Education de Stanford University, Institute of Education de University of London y Centre of Higher Education and Work de Kassel University y OISE-University of Toronto. Premio Konex 2016 Humanidades Diploma al Merito (Fundación KONEX). E-mail: griquelm@) filo.uba.ar

NATALIA HERGER: Doctora de la Universidad de Buenos Aires (UBA). Magíster en Diseño y Gestión de políticas y Programas Sociales (FLACSO). Licenciada en Ciencias de la Educación (UBA). Investigadora del Programa Educación, Economía y Trabajo del Instituto de Investigaciones en Ciencias de la Educación, Facultad de Filosofía y Letras (UBA). Auxiliar docente en las materias Trabajo y Mercado Laboral y Economía de la Educación de la carrera de Ciencias de la Educación (UBA).E-mail: nath@filo.uba.ar

ALEXANDER IVAN KODRIC: Magister en Economía Política con mención en Economía Argentina (FLACSO). Lic. en Economía (UBA). Doctorando de la Facultad de Ciencias Económicas de la UBA. Investigador en Formación Programa Educación, Economía y Trabajo (PEET), Instituto de Investigaciones en Ciencias de la Educación, Facultad de Filosofía y Letras (UBA). Docente de la Facultad de Ciencias Económicas de la UBA y en la Universidad Nacional de General Sarmiento. E-mail: alexkodric@yahoo.com.ar 